\title{
NOUVELLE
}

\section{Un nouveau rôle non métabolique de la glutamine synthétase au cours de l'angiogenèse}

Charlotte Dubois $^{1,2}$, Guy Eelen ${ }^{1}$, Peter Carmeliet ${ }^{1}$

\footnotetext{
${ }^{1}$ Laboratory of angiogenesis and vascular metabolism, University of Leuven, center for cancer biology, VIB, campus Gasthuisberg, 0\&N4, Herestraat 49-B912, B-3000, Leuven, Belgique.

${ }^{2}$ Adresse actuelle : Inserm U-1003, laboratoire d'excellence, canaux ioniques d'intérêt thérapeutique, équipe labellisée par la Ligue nationale contre le cancer, Université de Lille, rue Paul Langevin, 59656 Villeneuve d'Ascq Cedex, France. peter.carmeliet@kuleuven.vib.be
}

> L'angiogenèse est un processus physiologique de croissance de nouveaux vaisseaux sanguins à partir de vaisseaux préexistants, qui se déroule lors du développement embryonnaire et chez l'adulte. L'angiogenèse pathologique est une caractéristique du cancer et de diverses maladies ischémiques et inflammatoires [1]. Ces dernières années, de nombreux groupes de recherche ont tenté de comprendre les mécanismes moléculaires impliqués dans l'angiogenèse pathologique. Ces efforts ont conduit à la découverte d'un nombre croissant de molécules pro- et anti-angiogéniques, dont celles ciblant le VEGF (vascular endothelial growth factor), une molécule jouant un rôle central dans ce processus.

Si cette approche a été validée au début des années 2000 par l'utilisation en clinique du premier anticorps anti-VEGF puis d'inhibiteurs pharmacologiques de l'activité kinase de récepteurs impliqués dans l'angiogenèse, les études pré-cliniques et cliniques menées ces dernières années ont toutefois modéré

$(\rightarrow)$ Voir la Synthèse de Y. Gu et al., $\mathrm{m} / \mathrm{s}$ $n^{\circ} 4$, avril 2016, page 370

l'enthousiasme initial [2] $(\rightarrow)$.

$\varepsilon n$ effet, une efficacité insuffisante et le développement de résistance(s) aux traitements, ont conduit à l'émergence de nouvelles hypothèses de recherches [3].

Dans ce contexte, nous avons entrepris une caractérisation du métabolisme des cellules endothéliales [4], qui forment les vaisseaux sanguins, afin d'identifier de nouvelles cibles pour les approches anti-angiogéniques. La glycolyse $[5,6]$, l'oxydation des acides gras [7, 8] et, plus récemment, le métabolisme de la glutamine/asparagine [9] sont apparus comme des régulateurs clés du métabolisme de ces cellules capables d'influencer l'angiogenèse en conditions physiologique et physiopathologique, pouvant 

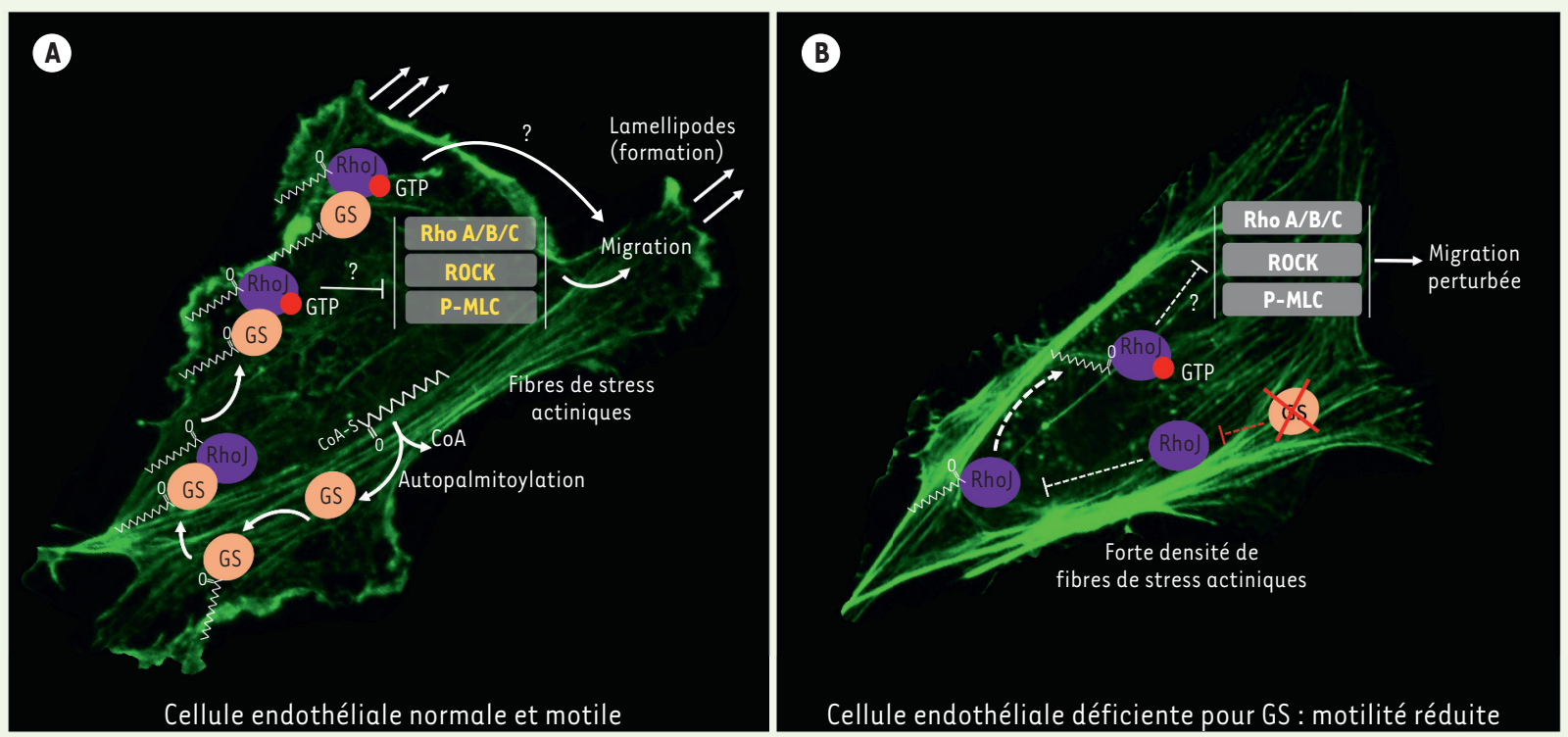

Figure 1. Rôle de la glutamine synthétase dans les cellules endothéliales. A. Dans les cellules endothéliales, la glutamine synthétase (GS) autopalmitoylée interagit directement (ou indirectement) avec la Rho GTPase RHOJ et maintient sa palmitoylation, sa localisation membranaire et l'activité de RHOJ (indiquées par la liaison au GTP). L'activité de RHOJ participe à la migration des cellules endothéliales, à la formation de lamellipodes et au contrôle de la formation de fibres de stress actiniques, ce qui favorise la migration des cellules endothéliales et la ramification des vaisseaux sanguins in vivo. Grâce à des mécanismes qui ne sont pas complètement compris, RHOJ activée inhibe la signalisation de la voie RHOA/B/C-ROCK(p)MLC (phospho-myosin light chain), elle-même connue pour favoriser la formation de fibres de stress. La contribution relative d'un effet direct de RHOJ sur la migration par rapport à l'effet indirect via RHOA/B /C- ROCK- (p) MLC reste encore à déterminer. RHOA/B/C, ROCK et (p)MLC sont en jaune pour indiquer une signalisation réduite de cette voie. $B$. La délétion de GS dans les cellules endothéliales rend RHOJ moins active, se traduisant par une diminution de sa palmitoylation et de sa liaison à la membrane, et réduit l'inhibition de la voie RHOA/B /C - ROCK- (p)MLC. La formation excessive de fibres de stress qui en résulte entraîne la perte de la capacité migratoire des cellules endothéliales, c'est-à-dire une réduction de la ramification des vaisseaux in vivo. Les lignes pointillées indiquent une activité réduite ; la croix rouge indique l'inhibition de GS; les points d'interrogation indiquent les mécanismes inconnus. L'actine est visualisée par marquage avec de la phalloïdine (fluorescence verte).

potentiellement être considérés comme de nouvelles cibles thérapeutiques.

Bien que le catabolisme de la glutamine (sa dégradation conduisant à la production d'énergie) dans les cellules endothéliales ait été récemment caractérisé [9], I'implication de son anabolisme (ensemble de réactions chimiques de synthèse moléculaire) dans le contrôle de l'angiogenèse in vivo restait méconnue. La glutamine est un donneur de carbone et d'azote nécessaire à la production de biomolécules. Elle participe également à l'homéostasie d'oxydoréduction (redox).

La plupart des cellules absorbent la glutamine et n'ont donc, elles-mêmes, pas besoin de la synthétiser. Pourtant, certains types de cellules expriment le gène
GLUL (glutamate-ammoniac ligase), codant la glutamine synthétase (GS), la seule enzyme capable de produire de novo de la glutamine à partir de glutamate et d'ammoniac, dans une réaction nécessitant de l'ATP (adénosine triphosphate) et du magnésium $\left(\mathrm{Mg}^{2+}\right)$ ou du manganèse $\left(\mathrm{Mn}^{2+}\right)$. La glutamine synthétase participe également à l'élimination de l'ammoniac, des effets qui sont bien décrits au niveau des hépatocytes, des astrocytes et des muscles.

Contre toute attente, notre étude [10] montre que la GS est exprimée par les cellules endothéliales qui sont pourtant, via la circulation sanguine, à proximité d'une importante concentration de glutamine $(0,6 \mathrm{mM})$. Nos données confirment qu'en effet à cette concentration de glutamine extracellulaire, les cellules endothéliales absorbent la glutamine et que la production de glutamine par la GS reste minimale. Néanmoins, en étudiant des souris génétiquement modifiées qui n'expriment pas glul spécifiquement dans les cellules endothéliales, nous avons montré des altérations vasculaires frappantes et une inhibition de l'angiogenèse au cours du développement.

Ces effets sont liés à l'inhibition de la migration des cellules endothéliales, et sont indépendants de leur prolifération. En effet, aucune perturbation de la prolifération (suivi par l'incorporation de $\varepsilon d U$ [5-éthynyle-2'-déoxyuridine] durant la synthèse de I'ADN dans les cellules endothéliales des vaisseaux 
sanguins de la rétine de l'œil) n'a pu être mise en évidence en comparant les souris contrôles et les souris déficientes pour glul spécifiquement dans les cellules endothéliales.

D'un point de vue mécanistique, nous avons démontré en utilisant différents types de cellules endothéliales (provenant de veines, artères, et de micro-vaisseaux) in vitro que la délétion du gène GLUL diminuait la localisation membranaire et l'activation de la GTPase RHOJ tout en activant d'autres Rho GTPases (RHOA/B/C) et la Rho kinase (ROCK), induisant ainsi la phosphorylation de la protéine MLC ( $p$-myosin light chain) et la formation de fibres de stress actiniques qui empêchent la motilité des cellules endothéliales.

Nous avons également découvert que la glutamine synthétase était capable de s'auto-palmitoyler, d'interagir avec la GTPase RHOJ et ainsi de favoriser la palmitoylation, l'ancrage à la membrane plasmique et l'activation de RHOJ. Localisée à la membrane plasmique et active, RHOJ va (de façon directe ou indirecte) diminuer la signalisation par la voie RHOA/B/C - ROCK - pMLC, ce qui contribue à l'intégrité du cytosquelette actinique de la cellule endothéliale et donc à sa motilité (Figure 1).
Ces découvertes révèlent ainsi un rôle pour l'enzyme GS qui est associé à une nouvelle activité de la protéine, impliquée dans la migration des cellules endothéliales en conditions physiologique et physiopathologique. Les cellules endothéliales ne migrent pas quand elles sont à l'état quiescent, ce qui est le cas chez l'adulte en bonne santé et explique pourquoi l'inhibition de la GS n'a pas d'effets observables sur le système vasculaire chez des souris adultes en bonne santé. Compte tenu de ces résultats, la GS représente une cible attrayante pour une inhibition thérapeutique de l'angiogenèse pathologique. Le MSO (methionine sulfoximine), un inhibiteur pharmacologique de la GS, est ainsi capable de réduire l'angiogenèse pathologique dans les cas de cécité et de psoriasis cutané, ce qui justifie une exploration plus approfondie du ciblage de cette enzyme pour des stratégies antiangiogéniques. $\diamond$

New non-metabolic role for glutamine synthetase in angiogenesis

\section{REMERCIEMENTS}

Les auteurs remercient l'ensemble du Laboratoire d'angiogenèse et de métabolisme vasculaire. Les recherches reçoivent le support du gouvernement fédéral de Belgique, du gouvernement
Flamand «long-term structural Methusalem», de la fondation FWO (Fonds voor Wetenschappelijk Onderzoek), de la fondation contre le cancer Belge, et du soutien européen avec « $E R C$ advanced research grant ».

\section{LIENS D'INTÉREิT}

Les auteurs déclarent n'avoir aucun lien d'intérêt concernant les données publiées dans cet article.

\section{RÉFÉRENCES}

1. Carmeliet P. Angiogenesis in health and disease. Nat Med $2003 ; 9: 653-60$.

2. Gu Y, Buisson-Vidal C, Li H, et al. La résistance aux traitements antiangiogéniques - Une activité clinique et scientifique. Med Sci (Paris) 2016 ; $32: 370-7$.

3. Carmeliet P, Jain RK. Principles and mechanisms of vessel normalization for cancer and other angiogenic diseases. Nat Rev Drug Discov 2011 ; 10 : 417-27.

4. Eelen $G$, de Zeeuw $P$, Treps $L$, et al. Endothelial cell metabolism. Physiol Rev $2018 ; 98$ : 3-58.

5. De Bock K, Georgiadou M, Schoors S, et al. Role of PFKFB3-driven glycolysis in vessel sprouting. Cell $2013 ; 154: 651-63$

6. Schoors S, De Bock K, Cantelmo AR, et al. Partial and transient reduction of glycolysis by PFKFB3 blockade reduces pathological angiogenesis. Cell Metab 2014 ; $19: 37-48$.

7. Bruning U, Morales-Rodriguez F, Kalucka J, et al. Impairment of angiogenesis by fatty acid synthase inhibition involves mTOR malonylation. Cell Metab $2018 ; 28: 866-80 . e 15$

8. Kalucka J, BierhansI L, Conchinha NV, et al. Quiescent endothelial cells upregulate fatty acid $\beta$-oxidation for vasculoprotection via redox homeostasis. Cell Metab $2018 ; 28$ : 881-94.el3.

9. Huang $\mathrm{H}$, Vandekeere $\mathrm{S}$, Kalucka J, et al. Role of glutamine and interlinked asparagine metabolism in vessel formation. EMBOJ 2017 ; 36 : 2334-52.

10. Eelen G, Dubois C, Cantelmo AR, et al. Role of glutamine synthetase in angiogenesis beyond glutamine synthesis. Nature $2018 ; 561: 63-9$.

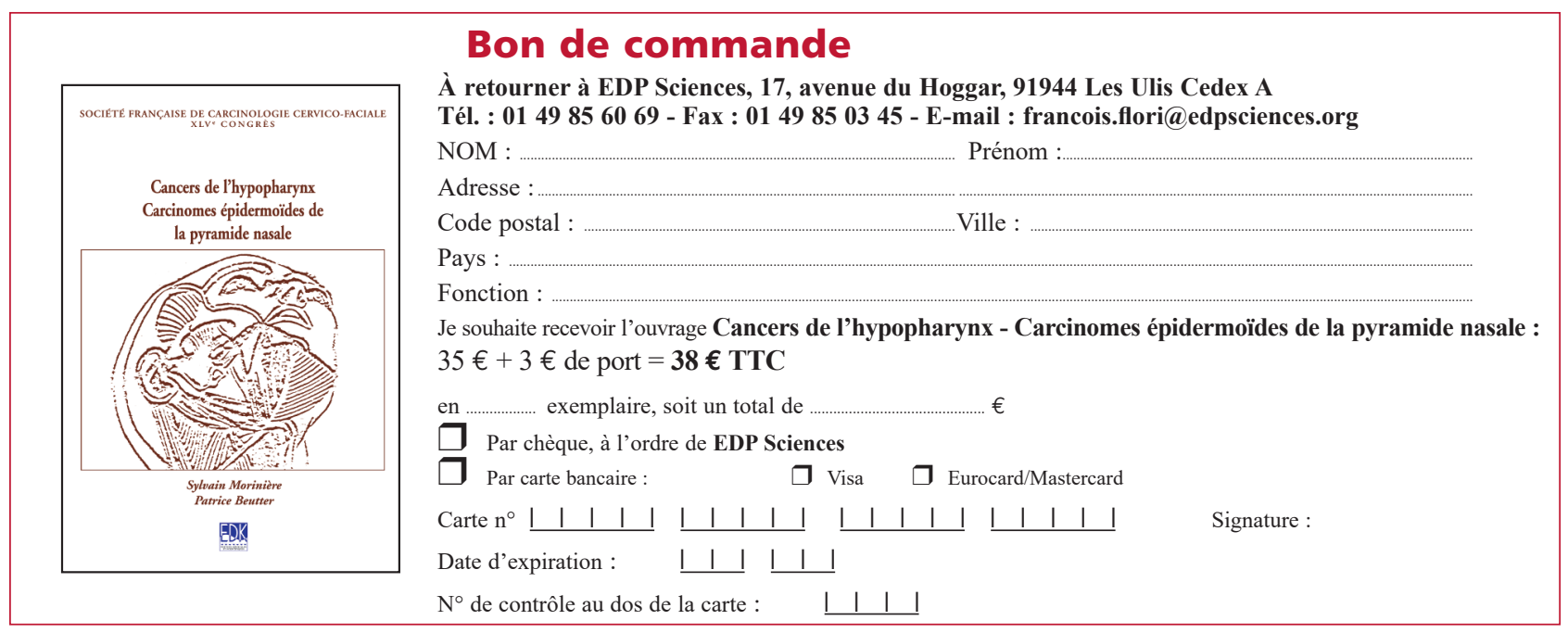

